\title{
Joining the dots from SNPs to proteins
}

A recent study provides the first genome-wide investigation of the relationship between common human genetic variation and levels of disease-related proteins. The findings have implications for understanding the aetiologies of complex diseases and how genetic variation influences the protein output of the genome.

Genome-wide association studies have made important inroads into understanding how common genetic variation influences complex disease. But the variants that have been identified generally have only small effects on the phenotypes of interest and often provide little insight into the functional basis of the association. A complementary strategy is to look for effects of genetic variation on gene expression. Although many variants that influence mRNA levels have been identified, the question remains of how such variation

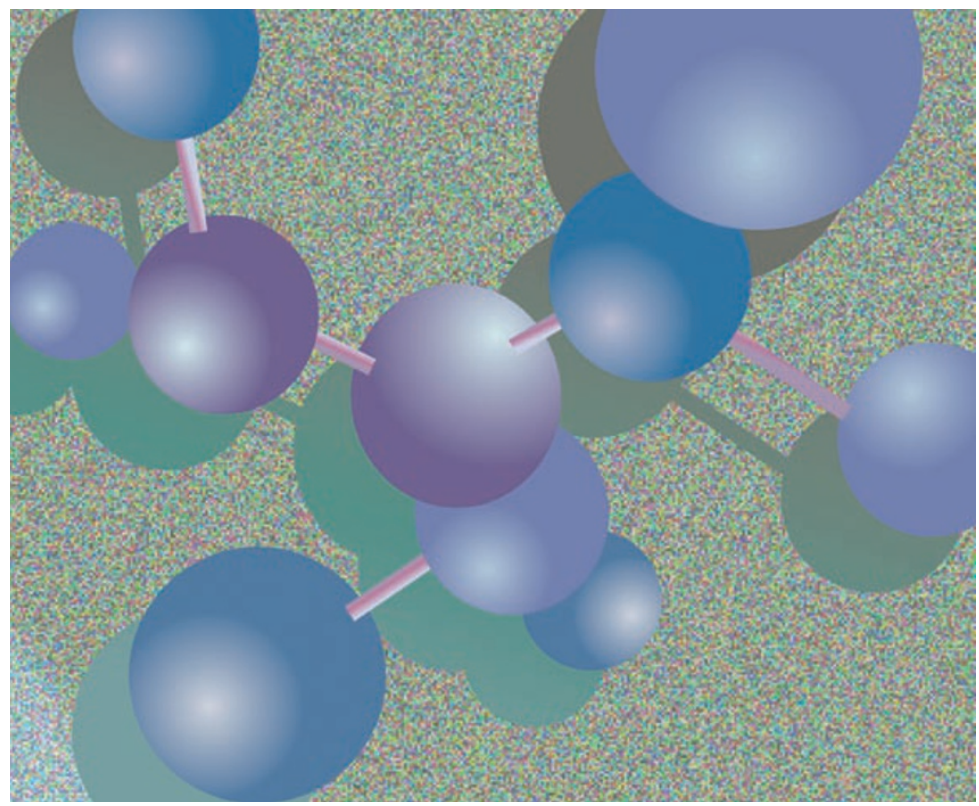
given the range of processes that affect protein abundance.

To begin answering this question, Melzer and colleagues looked for associations between almost half a million common SNPs and the blood levels of 42 proteins for which altered abundances in plasma or serum are correlated with disease status. The proteins chosen were relevant to a range of conditions, from metabolic and inflammatory disorders to HIV progression. Using samples from 1,200 individuals from the Italian InCHIANTI study, and applying stringent significance criteria, the authors identified 8 cis associations (that is, involving SNPs within 300 $\mathrm{kb}$ of the relevant gene). A single strong trans effect was also detected, but not replicated, possibly owing to differences in protein measurement between studies. ultimately influences proteins levels,

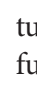
further studies to detect a wider range of effect sizes for a larger number of proteins. The results also have direct implications for understanding disease mechanisms, for example, in determining whether altered levels of certain proteins are causes or consequences of disease.

Louisa Flintoft

ORIGINAL RESEARCH PAPER Melzer, D. et al. A genome-wide association study identifies protein quantitative trait loci (pQTLs). PLoS Genet. 4, e1000072 (2008) FURTHER READING McCarthy, M. I. et al. Genome-wide association studies for complex traits: consensus, uncertainty and challenges. Nature Reviews Genet. 9, 356-369 (2008) 